\title{
Threatened Abortion
}

National Cancer Institute

\section{Source}

National Cancer Institute. Threatened Abortion. NCI Thesaurus. Code C112857.

Vaginal bleeding preceding the 20th week of gestation. 